\title{
Dynamic nuclear polarization by frequency modulation of a tunable gyrotron of $260 \mathrm{GHz}$
}

\author{
Dongyoung Yoon ${ }^{\mathrm{a}, *}$, Murari Soundararajan ${ }^{\mathrm{a}}$, Philippe Cuanillon ${ }^{\mathrm{a}}$, Falk Braunmueller ${ }^{\mathrm{b}}$, \\ Stefano Alberti ${ }^{\mathrm{b}}$, Jean-Philippe Ansermet ${ }^{\mathrm{a}}$ \\ ${ }^{a}$ École Polytechnique Fédérale de Lausanne, Institute of Condensed Matter Physics, CH-1015 Lausanne-EPFL, Switzerland \\ (email: dongyoung.yoon@epfl.ch, T: +41216934523) \\ ${ }^{b}$ Centre de Recherches en Physique des Plasmas, Station 13, École Polytechnique Fédérale de Lausanne, CH-1015 \\ Lausanne-EPFL, CH-1015 Lausanne, Switzerland
}

\begin{abstract}
An increase in Dynamic Nuclear Polarization (DNP) signal intensity is obtained with a tunable gyrotron producing frequency modulation around $260 \mathrm{GHz}$ at power levels less than $1 \mathrm{~W}$. The sweep rate of frequency modulation can reach $14 \mathrm{kHz}$, and its amplitude is fixed at $50 \mathrm{MHz}$. In water/glycerol glassy ice doped with $40 \mathrm{mM}$ TEMPOL, the relative increase in the DNP enhancement was obtained as a function of frequency-sweep rate for several temperatures. A $68 \%$ increase was obtained at $15 \mathrm{~K}$, thus giving a DNP enhancement of about 80 . By employing $\lambda / 4$ and $\lambda / 8$ polarizer mirrors, we transformed the polarization of the microwave beam from linear to circular, and achieved an increase in the enhancement by a factor of about $66 \%$ for a given power.
\end{abstract}

Keywords: DNP, TEMPOL, frequency modulation, gyrotron, millimeter waves, circular polarization

\section{Introduction}

Dynamic nuclear polarization (DNP) has been known for a long time to enhance nuclear spin polarization by transferring electron spin polarizations to nuclear spins. The enhancement $\varepsilon$ of nuclear spin polarization by DNP can in principle reach the value $\gamma_{e} / \gamma_{n}(\sim 660$ for ${ }^{1} \mathrm{H} \& \sim 2600$ for ${ }^{13} \mathrm{C}$ ), and DNP has been of great interest for improving the inherently low NMR signal to noise ratio (SNR) of low $\gamma$ nuclei[1]. However, there have been many obstacles to performing DNP in a magnetic field strong enough to obtain the high-resolution NMR

\footnotetext{
${ }^{*}$ Corresponding author
}

required for chemical or biological materials, because at these high fields, the electron paramagnetic resonance (EPR) falls in the TeraHertz $(\mathrm{THz}, 100$ $\mathrm{GHz}-10 \mathrm{THz}$ ) frequency range. As the technology in this frequency range has been rapidly improving, giving access to $\mathrm{THz}$ generators, waveguides, and vector network analyzers (VNA), recent DNPNMR studies have shown SNR improvement more than a 100 -fold at $9.4 \mathrm{~T}[2-5]$.

Gyrotrons used as microwave (MW) sources, for example in MAS-DNP, are generally monochromatic (typical natural width $\Delta f / f<100 \mathrm{kHz}$ ) and cannot depolarize all electron spins, even when there is spectral diffusion. In this paper, we show how frequency-modulation of the gyrotron improves the polarization transfer. In DNP studies with nitric oxide radicals at high magnetic field, solid state MW sources have been employed for 
frequency-modulated MW. Recently, Hovav et al. [6] have successfully reported an increase in the DNP enhancement by modulation using a solid state MW source at $3.34 \mathrm{~T}$ from $10 \mathrm{~K}$ to $50 \mathrm{~K}$. They showed the increase in $\varepsilon$ as a function of the amplitude $A_{m d}$ and the sweep rate $f_{m d}$ of modulation and obtained an increase in $\varepsilon$ by a factor of about 3. In their simulation, they considered the nuclear polarization enhanced by the solid effect (SE) and cross effect (CE) with frequency modulation, and showed that the number of active electrons contributing to DNP increases with increasing $A_{m . d}$ unless single quantum transitions are induced by off-resonance or spectral diffusion. They also showed that the nuclear polarization obtained by the solid effect is independent of $f_{m d}$, while that by the cross effect is effectively enhanced provided $f_{m d}$ is higher than the electron spin-lattice relaxation rate $1 / T_{1}^{e}$. Field modulation rather than frequency modulation was also shown to increase $\varepsilon$. Thurber et al. [7] performed DNP with field modulation at $9.4 \mathrm{~T}$. They showed that $\varepsilon$ can be increased by a factor of 2 at temperatures and radical concentrations suitable for the CE. $\varepsilon$ was shown to increase with increasing $f_{m d}$, up to the point when $f_{m d}$ exceeds the electron spin-lattice relaxation rate $1 / T_{1}^{e}$. In their simulation, they considered the cross effect and assumed that the spectral diffusion of electron spins is not fast enough to spread the depolarization across neighboring spin packets during $T_{1}^{e}$. Frequency modulation was also reported to increase DNP signal intensity in dissolution-DNP where DNP mechanism is thermal mixing[8]. Bornet et al. showed an increase in $\varepsilon$ with increasing $A_{m d}$ and $f_{m d}$, faster build-up rate, and more efficient cross-polarization. Besides nitric oxide radicals, silicon particles with defects on the surface have been previously used for DNP with frequency modulation[9].

These frequency modulation experiments with nitric oxide radicals reported so far were only at low temperatures, presumably because of the low power of solid state MW sources.

In this study, we present the enhancement of ${ }^{1} \mathrm{H}$ nuclear spin polarization by DNP for static samples in $9.28 \mathrm{~T}$ using a gyrotron MW source capable of frequency-tuning, and frequency-modulation, and ns pulsed regime[10, 11]. We obtained an increase of $\varepsilon$ by frequency modulations at fields higher than reported previously. Further more, as gyrotrons produce higher MW power than solid state MW sources, we were able to examine the effectiveness of frequency-modulation up to $100 \mathrm{~K}$. We also show that the conversion of linearly polarized MW to circular polarization improves $\varepsilon$ for a given MW power, as it has been shown with solid state MW sources and quasi-optical system[12, 13].

\section{Experiment set-up}

\subsection{Sample preparation}

We prepared samples by dissolving $40 \mathrm{mM}$ TEMPOL (Sigma Aldrich) in a mixture of $\mathrm{H}_{2} \mathrm{O}, \mathrm{D}_{2} \mathrm{O}$ and $\mathrm{d}_{8}$-glycerol of volume ratio 10: 30: 60 (DyNuPol. Inc). The sample of about $10 \mu \mathrm{l}$ was inserted in a sapphire tube of inner diameter $1 \mathrm{~mm}$, which has a high transmission for MW frequencies and a high thermal conductivity[14]. It is well known that glass-forming of samples composed of water and radicals is vital for high enhancement. We quenched the samples by inserting the DNP-NMR probe quickly into a cryostat set at a temperature much colder than the vitrification temperature. This implies a cooling rate higher than the minimum rate for vitrification of the sample, which is about $70 \mathrm{~K} / \mathrm{min}$ for $60 \%$ glycerol[15-17]. We observed by NMR that the sample solidification is generally completed in $1 \mathrm{~min}$, thus insuring glassformed samples.

\subsection{Frequency-tunable Gyrotron}

The main design features of the gyrotron prototype[10, 18, 19] are: frequency stability(with long-term feedback stabilization), spectral purity, fast and slow tunability, and MW-power control over a wide range. The cavity resonator and uptaper of the gyrotron were designed with special attention in order to achieve frequency-tunability in the vicinity of $260 \mathrm{GHz}$, relying on the smooth transition between axial eigenmodes of the cavity, as it has been reported on other papers about gyrotrons[20, 21]. A high flexibility of the electron beam parameters is achieved by making use of a triode-magnetron-injection gun with an independent control of the anode-voltage, allowing a fast frequency tunability. Furthermore, as described e.g. by Barnes et al.[20], a slow but wide range tunability is obtained by changing the cavity magnetic field and other control parameters.

The gyrotron characterization showed a maximum power of $150 \mathrm{~W}$ at a frequency of $f_{0}=260.45 \mathrm{GHz}$. 
The Vlasov converter together with the internal optical system ensures a high gaussian mode content of the microwave beam at the gyrotron-window[22]. The gyrotron output frequency, which can be longterm stabilized by a feedback-control[22], can be tuned on a time scale of several tens of seconds over a range of $\Delta f=1.2 \mathrm{GHz}$ with a power in excess of $1.5 \mathrm{~W}$. Fast tuning over a frequency range of $\sim 100 \mathrm{MHz}$ on a minimum time scale of $\sim 30 \mu \mathrm{s}$ can be achieved by the modulation of the anode voltage, as it has been described elsewhere using a modulation of the accelerating voltage $[23,24]$. This fast tuning is illustrated in Fig. 1, showing the frequency modulation by the anode voltage modulation. As we sweep the anode voltage in the gyrotron for frequency modulation with other parameters fixed, the MW frequency follows the anode voltage while the MW power is not constant in the range of $260.82 \pm 0.050 \mathrm{GHz}$; the highest power of about $1.6 \mathrm{~W}$ occurs at $260.8 \mathrm{GHz}$ and the power keeps decreasing down to about $0.4 \mathrm{~W}$ at the frequency maximum of the sweep.

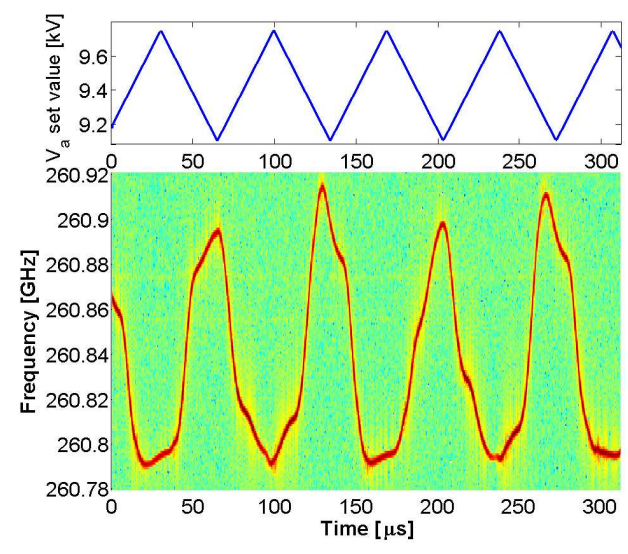

Figure 1. (Colour online) real-time MW-spectrum (bottom) and anode voltage control (top) with fast frequency modulation (sweep rate $f_{m d}$ and amplitude $A_{m d}$ of fast frequency modulation $=14.5 \mathrm{kHz}$ and $50 \mathrm{MHz}$, respectively).

The diagnostics which are used to analyze the MW-characteristics mainly consist of a calorimeter, a VDI heterodyne detection board (Virginia Diodes, Inc) connected to a $3 \mathrm{GHz}$-oscilloscope for measuring the instantaneous frequency and of a Schottky-diode for measuring the instantaneous power[10].

\subsection{Matching Optics Unit and attenuator}

A matching optical unit (MOU) (Fig. 2 (a)) is composed of a plane mirror, a $\lambda / 4$ polarizer mir- ror, a focusing mirror, and a $\lambda / 8$ polarizer mirror, which are designed for maximizing the coupling between the $\mathrm{HE}_{11}$ mode supported by the corrugated waveguide and the gaussian beam radiated from the gyrotron. The two corrugated polarizer mirrors in the MOU enable the transformation of the linearly polarized gyrotron output to a circular polarization or an elliptical polarization, after sequential reflections from the $\lambda / 4$ mirror and the $\lambda / 8$ mirrors $[25,26]$. The $\lambda / 4$ mirror is manufactured with metallic grooves of period $\mathrm{d}=0.500$ $\mathrm{mm}$, width $\mathrm{c}=0.250 \mathrm{~mm}$, and depth $\mathrm{h}=0.310$ $\mathrm{mm}$, and the $\lambda / 8$ mirror with the same $\mathrm{d}$ and $\mathrm{c}$, but $\mathrm{h}=0.214 \mathrm{~mm}[27]$. Based on the geometry shown in Fig. 2 (a), when the groove axis orientation angle $\phi_{\lambda / 4}$ of the $\lambda / 4$ mirror and the angle $\phi_{\lambda / 8}$ for the $\lambda / 8$ mirror are $49.1^{\circ}$ and $0^{\circ}$, respectively, with respect to linearly polarized incoming MW, the MW reflected off the mirrors maintains its polarization. When both angles are at $49.1^{\circ}$, the MOU converts the linear polarization to circular polarization. Therefore, linearly polarized MW is able to be transformed from $100 \%$ linear to 100 $\%$ circular polarization by rotating the $\phi_{\lambda / 8}$ from $0^{\circ}$ to $49.1^{\circ}$, while keeping the $\phi_{\lambda / 4}$ at $49.1^{\circ}$.

We used an attenuator (Tydex) to reduce MW power, which is composed of 5 silicon wafers with different transmissions $(70 \%, 30 \%, 10 \%, 3 \%$, and $1 \%)$. Combinations of the wafers in the attenuator enables us to adjust the MW power while keeping the gyrotron parameters constant. The attenuator is inserted between the entrance of the wave guide and the $\lambda / 8$ mirror, as shown in Fig. 2 (a).

\subsection{DNP-NMR probe and NMR measurement}

The NMR probe (Fig. 2 (b) \& (c)) is composed of a corrugated waveguide (Swissto12) and an NMR resonator circuit. The NMR coil is a solenoid of 4-6 turns with $1 \mathrm{~mm}$ spacing. It is placed close to the open end of the waveguide. Two variable capacitors are used for tuning and matching at ${ }^{1} \mathrm{H}$ NMR frequency $=395.2 \mathrm{MHz}$. The quality factor $\mathrm{Q}$ of the NMR resonator is about 60 . A demountable miter bend is used to couple the probe to the waveguide that brings the MW beam from the MOU to the NMR probe.

${ }^{1} \mathrm{H}$ NMR signal intensity is acquired by a saturation-recovery pulse sequence as described in Fig. 3. The saturation pulse, which is a comb pulse composed of a train of $90^{\circ}$ pulses, is used to set 
(a)

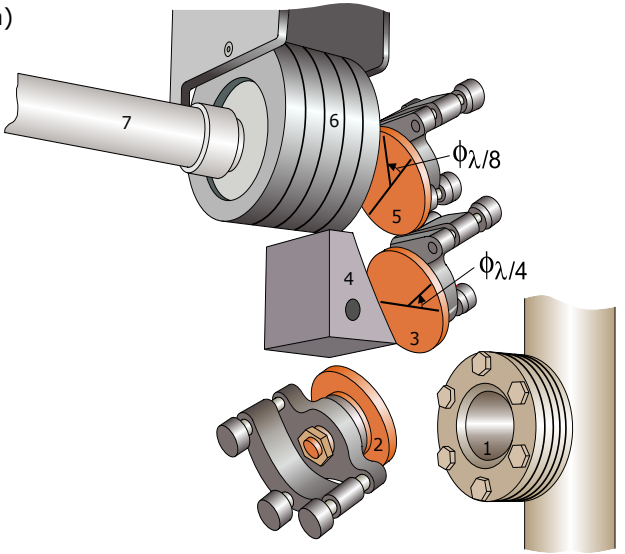

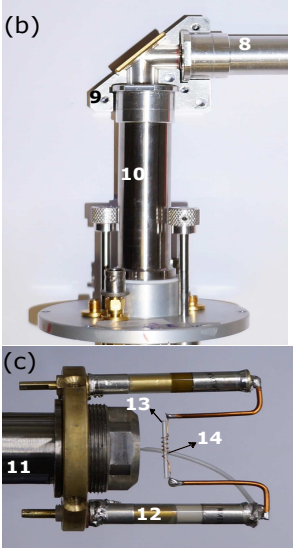

Figure 2.

(a) MOU: MW window (1) of the gyrotron, plane mirror (2), $\lambda / 4$ mirror (3), focusing mirror (4), $\lambda / 8$ mirror (5), attenuator (6) composed of 5 silicon wafers whose transmissions are $70 \%, 30 \%, 3 \%$, and $1 \%$, and waveguide (7). The short and long lines in the $\lambda / 4$ and $\lambda / 8$ mirrors (the radius and diameter of the disks, respectively) represent the groove axes and the electric field axis of MW, respectively. The relative angles between those two lines are defined as $\phi_{\lambda / 4}$ and $\phi_{\lambda / 8}$, respectively, (b) top of the NMR probe: waveguide (8) (same as (7)), side section of the miter bend (9), waveguide going to the NMR resonator circuit(10), (c) bottom of the NMR probe: waveguide (11) (same as (10)), variable capacitor (12), sapphire tube (13), solenoid coil (14).

a constant initial state of the nuclear polarization. After the saturation pulse, the nuclear polarization grows during the time $\tau$ with or without MW and is detected by a solid echo pulse sequence composed of $90_{x}^{\circ}-\tau_{\text {echo }}-90_{y}^{\circ}[7,28]$. Since our gyrotron is fastswitchable, MW is rapidly turned on at the end of the saturation comb pulse, and is turned off right before the solid echo pulse. A duty cycle of $25 \%$ is maintained to allow the sample to cool and return to the initial temperature before every acquisition. The NMR signal intensity is obtained by integrating the solid echo signal in the time domain. The $90^{\circ}$ pulse width is about $5 \mu \mathrm{sec}$ and $\tau_{\text {echo }}$ is 100 $\mu s e c$ at $70 \mathrm{~K}$ or $250 \mu \mathrm{sec}$ at $20 \mathrm{~K}$. Long $\tau_{\text {echo }}$ is used in this study to eliminate background signals not coming from the sample.

The enhancement $\varepsilon$ in this study is defined as the gain in nuclear polarization obtained with DNP at a temperature $\mathrm{T}$ normalized to the thermalequilibrium nuclear polarization at the same temperature. It can be expressed as :

$$
\varepsilon=\frac{S_{D N P}-S_{0}}{S_{0}}
$$

where $S_{D N P}$ and $S_{0}$ represent NMR signal intensities with and without MW. $S_{D N P}$ and $S_{0}$ are obtained with and without MW during the time $\tau_{b . d}$ allocated for letting the DNP signal build up. As the growth rate of $S_{D N P}$ with $\tau_{b . d}$ is known to be
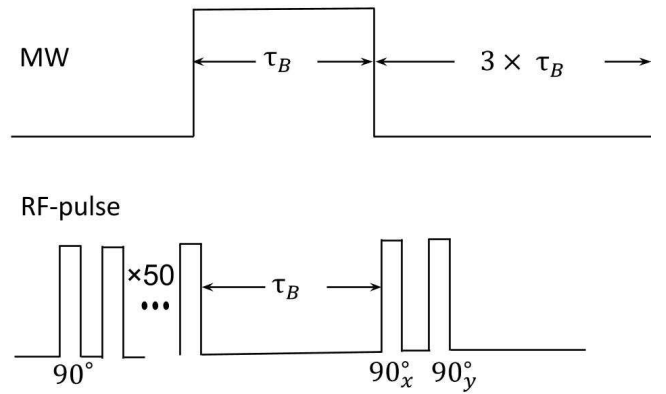

Figure 3. Solid echo $90_{\mathrm{x}}^{\circ}$ and $90_{\mathrm{y}}^{\circ}$ pulse sequence preceded by the saturation comb composed of $50-90^{\circ}$ pulses. The MW is turned on during $\tau_{b . d}$ and turned off during $\tau_{\text {off }}=3 \times \tau_{b . d}$.

similar to that of $S_{0}$ in the $\mathrm{CE}[6,7,29]$, we also obtained similar growth rates when MW power is attenuated to an appropriate level so as not to induce large dielectric heating of the sample. Therefore, we took the same $\tau_{b . d}=5 T_{1}^{n}$ for obtaining both $S_{D N P}$ and $S_{0}$, where $T_{1}^{n}$ is nuclear spin-lattice relaxation time without MW. 


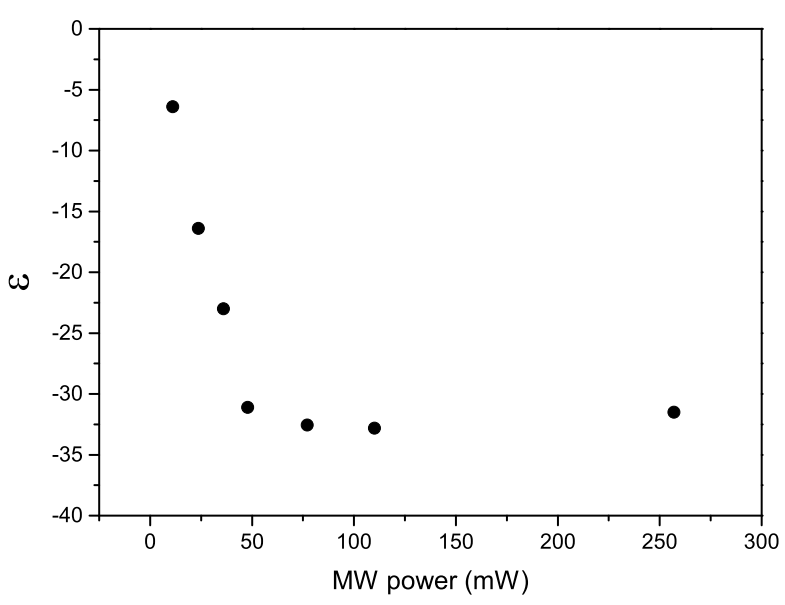

Figure 4. Enhancement $\varepsilon$ with $\mathrm{MW}$ power at $260.84 \mathrm{GHz}$ as a function of MW power at $20 \mathrm{~K}$ with linear polarization. The MW power is measured at the gyrotron window, and adjusted using the attenuator.

\section{Experimental result}

\subsection{Enhancement versus $M W$ polarization}

If MW is linearly polarized, it can be decomposed into clockwise and counterclockwise circular polarizations, and magnetic resonance will occur by absorbing only one appropriate handedness, while the other handedness does not contribute to the magnetic resonance. Thus, in principle, circular polarization with appropriate handedness can increase the effective $B_{1}$ field of MW by a factor of 2. Armstrong et al. reported previously that DNP signal intensity can be increased by $28 \%$ with the same MW power by transforming linearly-polarized MW to circularly-polarized MW using their quasi-optical units[13]. Thurber et al. also showed that $\varepsilon$ can be increased by about $25 \%$ by going from linear to circular polarization[12].

In order to show the polarization dependence of $\varepsilon$, we attempted to exclude the effect of dielectric heating on $\varepsilon$ by attenuating the MW power to a certain low level, which is in a range that $\varepsilon$ is proportional to MW power. Therefore, we obtained the DNP signal at $260.83 \mathrm{GHz}$ as a function of the MW power. It shows that $\varepsilon$ increases rapidly with increasing MW power, and reaches an asymptote above $70 \mathrm{~mW}$. Thus, we measured $\varepsilon$ as a function of $\phi_{\lambda / 8}$ with $10 \mathrm{~mW}$ (Fig. 5).

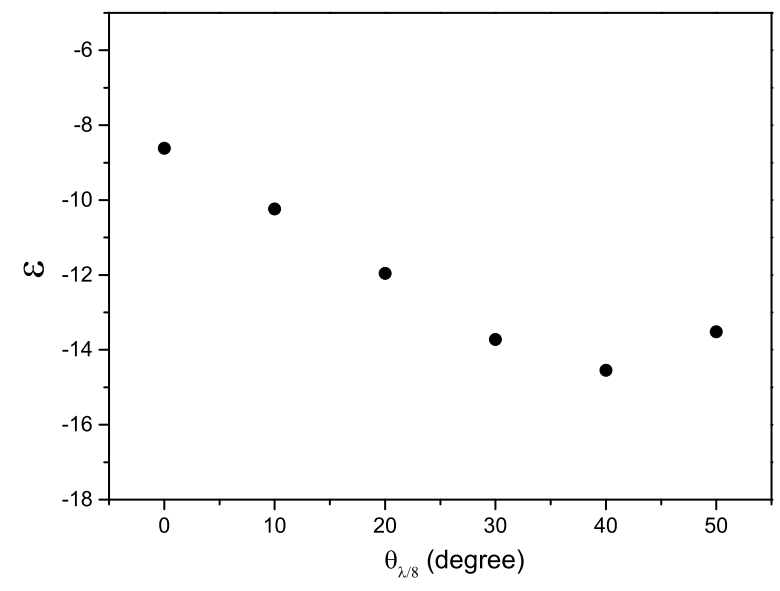

Figure 5. Enhancement $\varepsilon$ at $260.84 \mathrm{GHz}$ as a function of $\phi_{\lambda / 8}$ from $0^{\circ}$ to $49.1^{\circ}$ at $20 \mathrm{~K}$. The MW power is about 10 $\mathrm{mW}$ at all angles.

We set the $\theta_{\lambda / 8}$ at positive values to produce elliptical or circular polarization. We find that the enhancement changes by as much as $60 \%$ as we move away from the linear polarization. The maximum in $\varepsilon$ occurs at $40^{\circ}$. This value is different by about $10^{\circ}$ from our expectation, possibly due to practical imperfections, such as the misalignment of the mirrors, and the MW scattering in the NMR coil.

\subsection{DNP by frequency modulation}

Here, we present an increase in the enhancement $\varepsilon$ by frequency-modulated MW produced by the gyrotron, as shown in Fig. 6. Figure 7 shows the DNP frequency profile, which shows the enhancement versus MW frequency, obtained by tuning the MW frequency at fixed magnetic field. The maximum negative enhancement occurs about $260.7 \mathrm{GHz}$ and zero enhancement is found at $260.55 \mathrm{GHz}$, which is close to the center of the EPR spectrum. From Fig. 7, we chose $260.84 \mathrm{GHz}$ as the center frequency for the frequency modulation because this frequency-swept band is close to where the maximum enhancement occurs, so the electron spin packets within the amplitude $A_{m d}$ of frequency modulation and neighboring packets saturated by spectral diffusion also can contribute to the enhancement. The $A_{m d}$ is $50 \mathrm{MHz}$, and the sweep rates $f_{m d}$ of frequency modulation are 770 $\mathrm{Hz}, 2.3 \mathrm{kHz}, 6.3 \mathrm{kHz}$, and $14 \mathrm{kHz}$. As depicted 


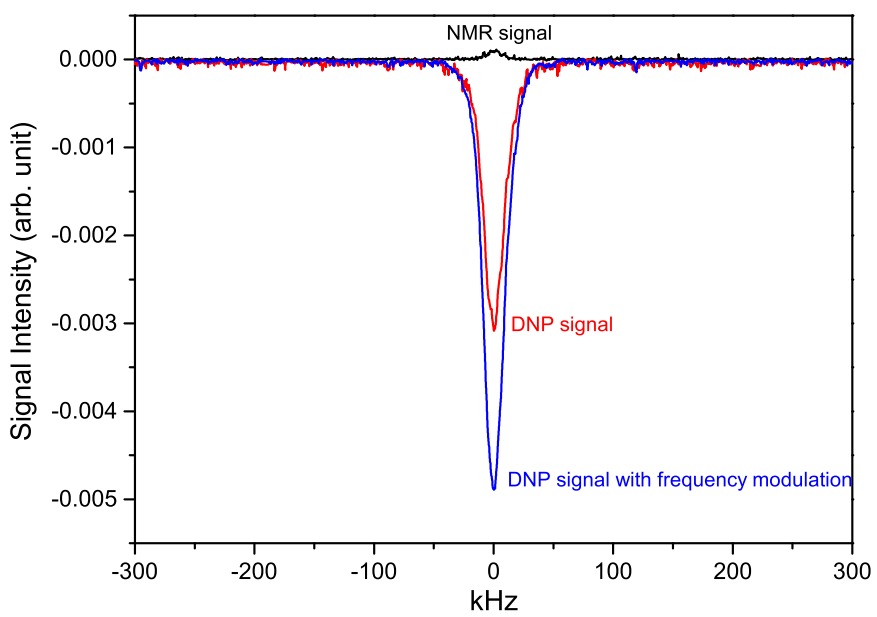

Figure 6. (Colour online $)^{1} \mathrm{H}$ NMR spectrum at $20 \mathrm{~K}$ without MW (top, black), with monochromatic MW at 260.84 $\mathrm{GHz}$ (middle, red), and with frequency-modulated $(14 \mathrm{kHz})$ MW (bottom, blue). The power levels of monochromatic and frequency-modulated $\mathrm{MW}$ are about $70 \mathrm{~mW}$. The center frequency of frequency-modulated MW is coincident with the monochromatic MW.

in Fig. 1, the modulation as a function of time is nearly triangular in shape.

Figure 8 shows $\varepsilon$ obtained by monochromatic MW and frequency-modulated MW at temperatures from $15 \mathrm{~K}$ to $100 \mathrm{~K}$. Since MW power with frequency modulation is $10 \%$ larger than that without frequency modulation, we used a large enough MW power at each temperatures to reach the maximum DNP signal in order to obtain enhancements independent of these changes of power levels. This $10 \%$ difference of the MW power is not expected to induce a significant change in dielectric heating of the sample. In Fig. $8, \varepsilon$ shows initially a rapid increase with $f_{m d}$, but the increase rate becomes smaller with increasing $f_{m d}$, as reported by others $[6,8]$. The increase of $\varepsilon$ by frequency modulation is defined as the gain $\eta$ described by,

$$
\eta(\%)=100 \times \frac{\varepsilon\left(f_{m d}\right)-\varepsilon(0)}{\varepsilon(0)},
$$

where $\varepsilon\left(f_{m d}\right)$ and $\varepsilon(0)$ are the enhancements with and without frequency modulation, respectively. We find that we were able to obtain a significant

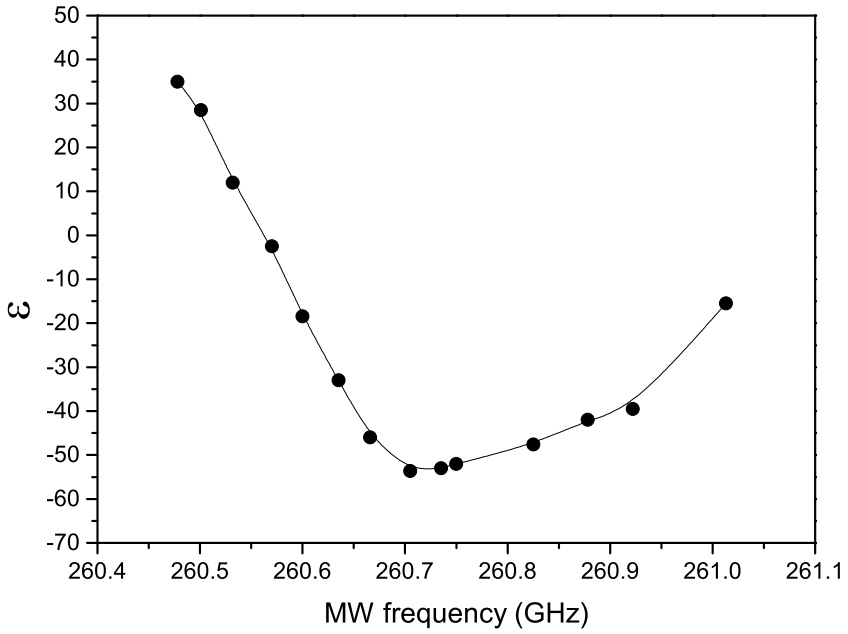

Figure 7. DNP frequency profile at $20 \mathrm{~K}: \varepsilon$ as a function of MW frequency at $20 \mathrm{~K}$ with MW power of $70 \mathrm{~mW}$. The DNP signal and NMR signals are obtained during the build-up time of $150 \mathrm{sec}\left(T_{1}^{n} \sim 30 \mathrm{sec}\right)$ with and without MW, respectively. DNP spectrum in the range of positive enhancements is uncompleted because the minimum tuning frequency of the gyrotron is $260.48 \mathrm{GHz}$.

increase $\eta$ with $f_{m d}=14 \mathrm{kHz}$ even at $100 \mathrm{~K}$, although $\varepsilon$ is small with the MW power of about 1 $\mathrm{W}$ at this temperature perhaps due to a high electron spin-lattice relaxation rate $1 / T_{1}^{e}$.

Our results give some information about the spin lattice relaxation time of electron spins $T_{1}^{e}$ by assuming that $\eta$ saturates if $f_{m d}$ exceeds over $1 / T_{1}^{e}$. As shown in [6], the polarization of nuclei near electrons oscillates if the frequency sweep rate is low, $f_{m d}<1 / T_{1}^{e}$. If the sweep rate is high, $f_{m d}>1 / T_{1}^{e}$, this polarization is constant, and the polarization of bulk nuclei is greater. We assume that the sweep rate, $f_{m d} \backsim 10 / T_{1}^{e}$, is fast enough to average these oscillations. To show the growth of $\eta$ with $f_{m d}$ more clearly, we show (Fig. 9) $\eta$ normalized to $\eta(14 \mathrm{kHz})$, for several temperatures. $\eta$ at $15 \mathrm{~K}$ and $20 \mathrm{~K}$ are shown to be almost at the maximum even for $f_{m d}$ as low as $2.3 \mathrm{kHz}$, which can be explained by assuming that $2.3 \mathrm{kHz}$ is faster than that electron spin relaxation rate $1 / T_{1}^{e}$ at these temperatures. Values of $\eta$ below $70 \mathrm{~K}$ are seen to saturate at $14 \mathrm{kHz}$, while $\eta$ at $100 \mathrm{~K}$ seems to still grow even beyond $14 \mathrm{kHz}$, indicating a much shorter $T_{1}^{e}$. Therefore, we expect that $T_{1}^{e}$ is of about a few msec at temperatures below $20 \mathrm{~K}$, and is shorter than $700 \mu s e c$ at $100 \mathrm{~K}$. By following the previously reported temperature and 


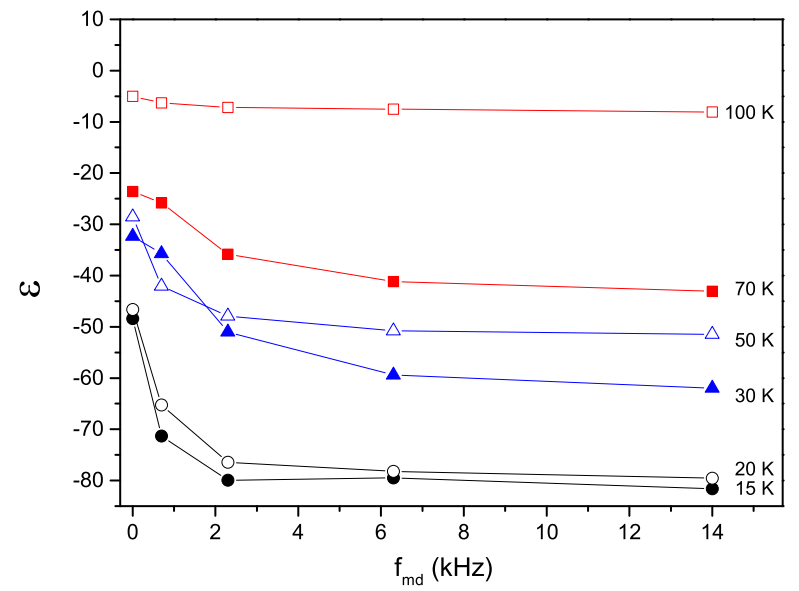

Figure 8. (Colour online)The $\varepsilon$ with and without frequency modulation at temperatures of $15 \mathrm{~K}$ (closed circle), 20 $\mathrm{K}$ (open circle), $30 \mathrm{~K}$ (closed triangle), $50 \mathrm{~K}$ (open triangle), $70 \mathrm{~K}$ (closed square), and $100 \mathrm{~K}$ (open square). The MW power levels at the sample position are about $70 \mathrm{~mW}$ at $15 \mathrm{~K}$ and $20 \mathrm{~K}, 153 \mathrm{~mW}$ at $30 \mathrm{~K}, 470 \mathrm{~mW}$ at $50 \mathrm{~K}, 720$ $\mathrm{mW}$ at $70 \mathrm{~K}$, and $1 \mathrm{~W}$ at $100 \mathrm{~K}$.

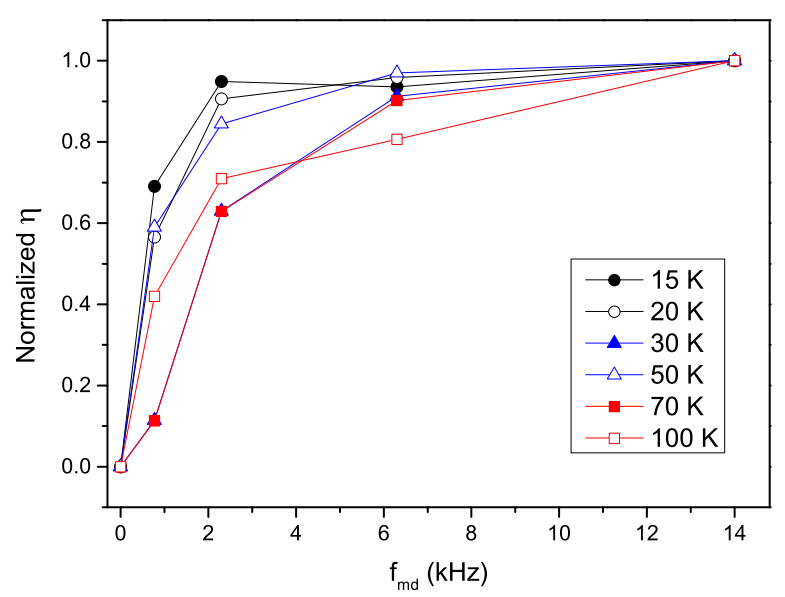

Figure 9. (Colour online)Normalized $\eta$ to $\eta(14 \mathrm{kHz})$ as a function of $f_{m d}$.

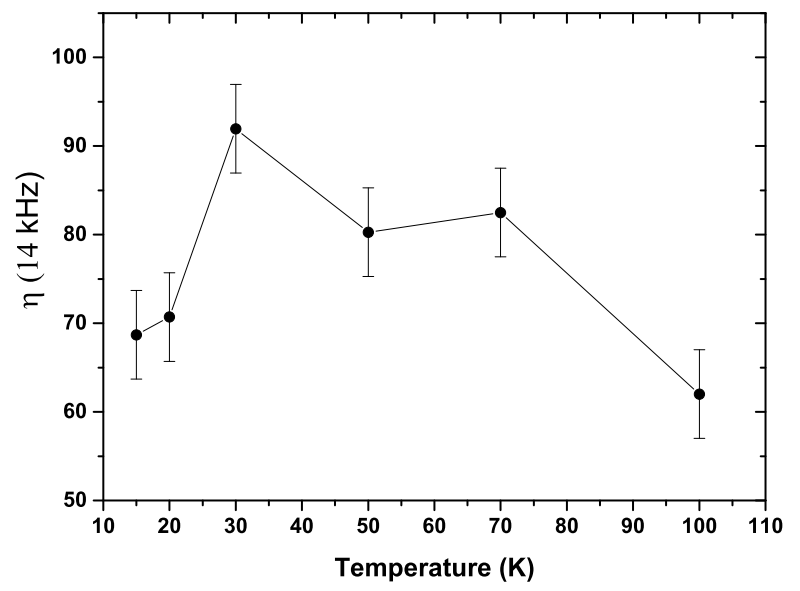

Figure 10. $\eta(14 \mathrm{kHz})$ as a function of temperature.

magnetic field dependence of $1 / T_{1}^{e} \propto B_{0}^{4} T[7,30-$ 32], we roughly estimated $T_{1}^{e} \simeq 5 \mathrm{msec}$ at $15 \mathrm{~K}$ and $T_{1}^{e} \simeq 750 \mu \mathrm{sec}$ at $100 \mathrm{~K}$, which is consistent with the values estimated from Fig. 9. These values of $1 / T_{1}^{e}$ are also approximately consistent with those previously reported[28, 33]. This also supports the assumption that the drop in $\varepsilon$ at $100 \mathrm{~K}$ is due to a small MW $B_{1}$ field in comparison with $1 / T_{1}^{e}$. Thus, a high $\varepsilon$ at $100 \mathrm{~K}$ requires high $B_{1}$ field overcoming $T_{1}^{e}$ or a $f_{m d}$ whose period is much shorter than $T_{1}^{e}$. In this study, however, we were unable to increase the MW power at $100 \mathrm{~K}$ beyond $1 \mathrm{~W}$ as our cooling power was not sufficient to overcome any higher dielectric heating.

The temperature dependence of $\eta(14 \mathrm{kHz})$ is depicted in Fig. 10. $\eta(14 \mathrm{kHz})$ increases from about $68 \%$ to about $92 \%$ with increasing temperature from $15 \mathrm{~K}$ to $30 \mathrm{~K}$, but decreases with further increase in temperature. However, $\eta(14 \mathrm{kHz})$ remains above $60 \%$ even at $100 \mathrm{~K}$, which might be interesting to those who use MAS-DNP at $100 \mathrm{~K}$. The temperature dependence of $\eta(14 \mathrm{kHz})$, which has a maximum at $30 \mathrm{~K}$, is consistent with that obtained at $95 \mathrm{GHz}[6]$. In the paper, $\eta$ increases with increasing temperature up to $50 \mathrm{~K}$. Further studies, such as temperature dependence of spectral diffusion rate and $T_{1}^{e}$, are required to understand the temperature feature. 


\section{Conclusion}

In this study, we have shown DNP obtained using a fast frequency-tunable, amplitude-switchable 260 $\mathrm{GHz}$ gyrotron with $40 \mathrm{mM}$ TEMPOL, at various temperatures. We obtained a DNP frequency profile by a step-wise sweep of the MW frequency over $500 \mathrm{MHz}$, at fixed NMR frequency. By sweeping the anode voltage of the gyrotron, we were able to perform a frequency modulation at sweep rates $f_{m d}$ up to $14 \mathrm{kHz}$, covering a frequency band of electron spin resonance of $100 \mathrm{MHz}$. Frequency modulation was shown to be effective in producing a gain in enhancement of more than $60 \%$ from $15 \mathrm{~K}$ to $100 \mathrm{~K}$. Thanks to the high power of the gyrotron, we were also able to show the effectiveness of the frequency modulation even at $100 \mathrm{~K}$. We achieved a small enhancement of about -8 and -5 with and without frequency modulation, respectively, at 100 $K$ because the enhancement saturates after about $1 \mathrm{~W}$ probably due to our poor cooling power. However, the effectiveness of frequency modulation at high temperature, even at $100 \mathrm{~K}$, would be still valuable to those who are able to apply high power frequency-modulated MW without much dielectric sample heating, as in MAS-DNP.

\section{Acknowledgements}

We gratefully acknowledge financial support by the Swiss National Science Foundation, Requip (No. 206021-121303/1), Sinergia (CRSI20$122708 / 1), F N\left(200021 \_153230\right)$, and by the École Polytechnique Fédérale de Lausanne (EPFL) and its Faculty of Basic Sciences.

[1] A. Abragam, Principles of Nuclear Magnetism, INTERNATIONAL SERIES OF MONOGRAPHS ON PHYSICS:32, OXFORD UNIVERSITY PRESS, 1960.

[2] P. Neugebauer, J. G. Krummenacker, V. P. Denysenkov, G. Parigi, C. Luchinat, T. F. Prisner, Phys. Chem. Chem. Phys. 15 (16) (2013) 6049-56.

[3] V. Bajaj, C. Farrar, M. Hornstein, I. Mastovsky, J. Vieregg, J. Bryant, B. Eléna, K. Kreischer, R. Temkin, R. Griffin, J. Magn. Reson. 160 (2003) 8590

[4] A. Zagdoun, G. Casano, O. Ouari, M. Schwarzwa, A. J. Rossini, F. Aussenac, M. Yulikov, G. Jeschke, C. Copéret, A. Lesage, P. Tordo, L. Emsley, J. Am. Chem. Soc. 135 (2013) 12790-12797.

[5] R. G. Griffin, T. F. Prisner, P. Chem, C. Phys, R. I. Hunter, P. A. S. Cruickshank, D. R. Bolton, P. C. Riedi, G. M. Smith, P. C. Chem, E. V. Kryukov, M. E. Newton, K. J. Pike, R. David, R. M. Kowalczyk, A. P. Howes, E. Mark, R. Dupree, S. Bowen, C. Hilty, K. R. Thurber, R. Tycko, Phys. Chem. Chem. Phys. 12 (2010) 5850.
[6] Y. Hovav, A. Feintuch, S. Vega, D. Goldfarb, J. Magn. Reson. 238 (2014) 94-105.

[7] K. R. Thurber, W.-M. Yau, R. Tycko, J. Magn. Reson. 204 (2010) 303-313.

[8] A. Bornet, J. Milani, B. Vuichoud, A. J. Perez Linde, G. Bodenhausen, S. Jannin, Chem. Phys. Lett. 602 (2014) 63-67.

[9] M. C. Cassidy, H. R. Chan, B. D. Ross, P. K. Bhattacharya, C. M. Marcus, Nat. Nanotechnol. 8 (5) (2013) 363-8.

[10] S. Alberti, J.-P. Ansermet, K. A. Avramides, F. Braunmueller, P. Cuanillon, J. Dubray, D. Fasel, J.-P. Hogge, A. Macor, E. de Rijk, M. da Silva, M. Q. Tran, T. M. Tran, Q. Vuillemin, Phys. Plasmas 19 (2012) 123102.

[11] S. Alberti, F. Braunmueller, T. M. Tran, J. Genoud, J.-P. Hogge, M. Q. Tran, J.-P. Ansermet, Phys. Rev. Lett. 111 (2013) 205101.

[12] K. R. Thurber, A. Potapov, W.-M. Yau, R. Tycko, J. Magn. Reson. 226 (2013) 100-106.

[13] B. D. Armstrong, D. T. Edwards, R. J. Wylde, S. A. Walker, S. Han, Phys. Chem. Chem. Phys. 12 (2010) 5920-5926.

[14] E. a. Nanni, A. B. Barnes, Y. Matsuki, P. P. Woskov, B. Corzilius, R. G. Griffin, R. J. Temkin, J. Magn. Reson 210 (1) (2011) 16-23.

[15] A. Baudot, L. Alger, P. Boutron, CRYOBIOLOGY 40 (2000) $151-158$.

[16] D. Banerjee, S. Bhat, J. Non-Cryst. Solids 355 (2009) 2433-2438.

[17] S. Bhat, A. Sharma, S. Bhat, Phys. Rev. Lett. 95 (2005) 235702.

[18] S. Alberti, J.-P. Ansermet, K. Avramides, D. Fasel, J.P. Hogge, S. Kern, C. Lievin, Y. Liu, A. Macor, I. Pagonakis, M. Silva, M. Tran, T. Tran, D. Wagner, in: 34th International Conference on Infrared, Millimeter, and Terahertz Waves, 2009, pp. $1-2$.

[19] Y. Rozier, F. Legrand, C. Lievin, J.-C. Racamier, R. Marchesin, S. Alberti, F. Braunmueller, J.-P. Hogge, M. da Silva, M. Q. Tran, T. M. Tran, A. Macor, in: 14th International Vacuum Electronics Conference (IVEC), 2013.

[20] A. B. Barnes, E. A. Nanni, J. Herzfeld, R. G. Griffin, R. J. Temkin, J. Magn. Reson. 221 (2012) 147-153.

[21] A. C. Torrezan, S.-T. Han, I. Mastovsky, M. a. Shapiro, J. R. Sirigiri, R. J. Temkin, A. B. Barnes, R. G. Griffin, IEEE Trans. Plasma Sci. 38 (6) (2010) 1150-1160.

[22] J.-P. Hogge, F. Braunmueller, S. Alberti, J. Genoud, T. Tran, Q. Vuillemin, M. Tran, J.-P. Ansermet, P. Cuanillon, A. Macor, E. de Rijk, P. Saraiva, in: 38th International Conference on Infrared, Millimeter, and Terahertz Waves, 2013, pp. 1-2.

[23] T. Idehara, E. M. Khutoryan, Y. Tatematsu, Y. Yamaguchi, a. N. Kuleshov, O. Dumbrajs, Y. Matsuki, T. Fujiwara, J Infrared Milli Terahz Waves 36 (9) (2015) 819-829.

[24] D. E. Hoff, B. J. Albert, E. P. Saliba, F. J. Scott, E. J. Choi, M. Mardini, A. B. Barnes, Solid State Nucl. Magn. Reson. (2015) http://dx.doi.org/10.1016/j.ssnmr.2015.10.001.

[25] A. V. Bieren, A. Macor, E. D. Rijk, J.-P. Ansermet, in: 4th International DNP Symposium, 2013.

[26] D. Wagner, F. Leuterer, INT J INFRARED MILLI 26 (2005) 163-172.

[27] E. J. D. RIJK, Terahertz passive components for dynamic nuclear polarization nuclear magnetic reso- 
nance applications, Ph.D. thesis, École Polytechnique Fédérale de Lausanne (2013)

[28] T. A. Siaw, M. Fehr, A. Lund, A. Latimer, S. a. Walker, D. T. Edwards, S.-I. Han, Phys. Chem. Chem. Phys. 16 (35) (2014) 18694.

[29] D. S. Wollan, Phys. Rev. B 13 (1976) 3671-3685.

[30] H. Sato, V. Kathirvelu, G. Spagnol, S. Rajca, A. Rajca, S. S. Eaton, G. R. Eaton, J. Phys. Chem. B. 112 (2008) 2818-2828.

[31] R. D. L. Kronig, Physica 6 (1) (1939) 33-43.

[32] P. L. Scott, C. D. Jeffries, Phys. Rev. 127 (1962) 32.

[33] B. Corzilius, L. B. Andreas, A. A. Smith, Q. Z. Ni, R. G. Griffin, J. Magn. Reson. 240 (2014) 113-123. 


\section{Gyrotron}

DNP SIGNAL

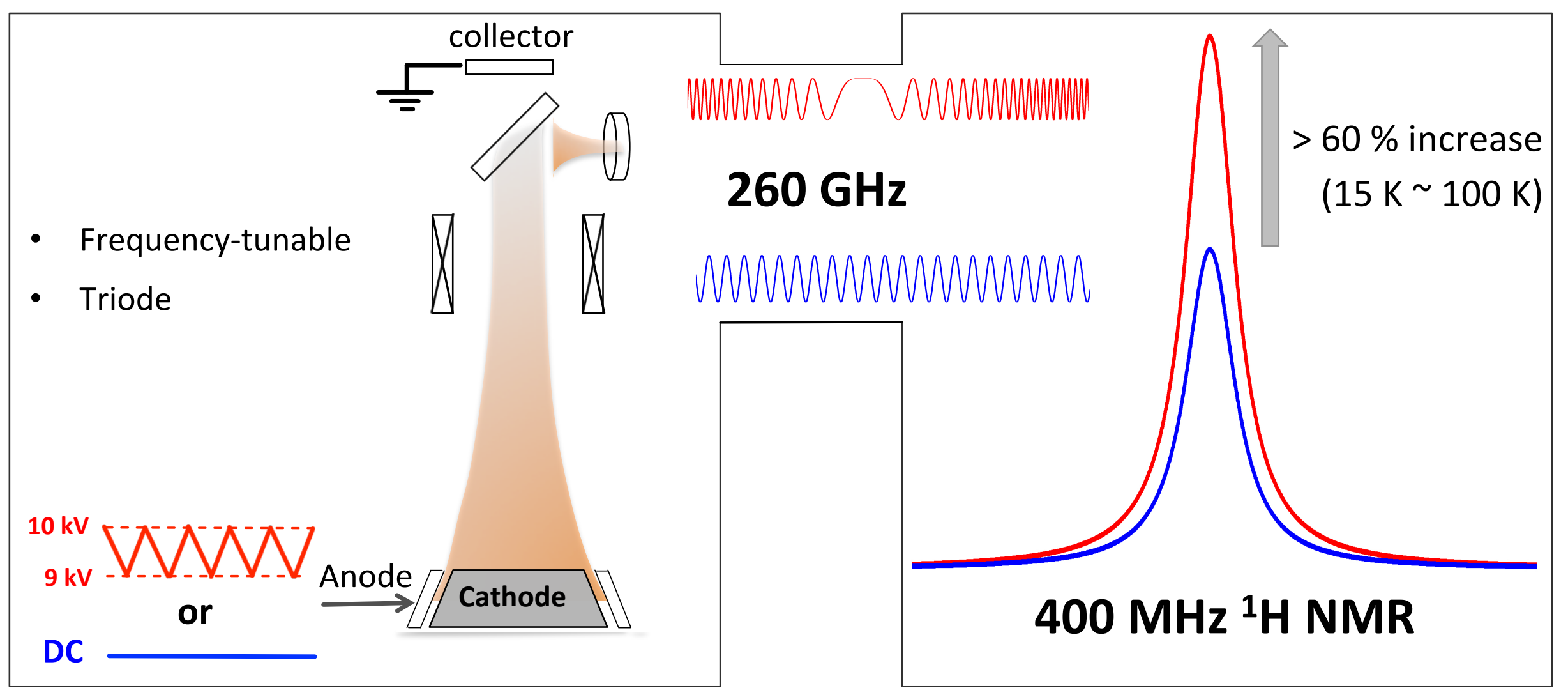

